Proceedings of the 2005 Winter Simulation Conference

M. E. Kuhl, N. M. Steiger, F. B. Armstrong, and J. A. Joines, eds.

\title{
FINDING THE BEST IN THE PRESENCE OF A STOCHASTIC CONSTRAINT
}

\author{
Sigrún Andradóttir \\ David Goldsman \\ Seong-Hee Kim \\ School of Industrial and Systems Engineering \\ Georgia Institute of Technology \\ Atlanta, GA 30332-0205, U.S.A.
}

\begin{abstract}
Our problem is that of finding the best system-i.e., the system with the largest or smallest primary performance measure - among a finite number of simulated systems in the presence of a stochastic constraint on a secondary performance measure. In order to solve this problem, we first find a set that contains only feasible or near-feasible systems (Phase I) and then choose the best among those systems in the set (Phase II). We present a statistically valid procedure for Phase I and then propose another procedure that performs Phases I and II sequentially to find the best feasible system. Finally, we provide some experimental results for the second procedure.
\end{abstract}

\section{INTRODUCTION}

Our goal is to select the best or near-best system from a set of competing systems, where the term "best" is with respect to a primary performance measure among the systems, which we want to maximize or minimize. We also want the selected system to satisfy a stochastic constraint on a secondary performance measure.

Due to randomness in output data, one needs to be careful when comparing a number of simulated systems. Over the last decade, there have been fruitful efforts in developing statistically valid ranking and selection (R\&S) procedures that find the best among a finite number of simulated alternatives. Boesel et al. (2003), Nelson et al. (2001), Kim and Nelson (2001, 2005), Goldsman et al. (2000, 2002), Chen (1996), Chen et al. (1997, 2000), Chick (1997), and Chick and Inoue (2001a, 2001b) have all developed different types of statistically valid or heuristic selection procedures that are useful in simulation when the goal is to find a system with the minimum or maximum expected performance measure among a finite number of simulated systems. They showed that their procedures are a great deal more efficient than classical R\&S procedures such as that due to Rinott (1978). However, a limitation of those procedures lies in the fact that they consider only one performance measure. In reality, we often face constraints on performance measures other than a primary performance measure due to physical or managerial limits placed on a system. When a stochastic constraint is present, existing $\mathrm{R} \& S$ procedures lose their statistical guarantee about a correct selection, defeating the biggest advantage of using $\mathrm{R} \& \mathrm{~S}$ procedures over heuristic procedures.

There is not a rich literature on solving discrete optimization problems with stochastic constraints using simulation. Butler et al. (2001) combined multiple attribute utility theory from economics with the Rinott (1978) procedure to handle multiple performance measures. Their procedure finds a system that gives the highest utility. But it does not handle constraints, and finding an appropriate attribute utility function itself is a very difficult problem. Santner and Tamhane (1984) proposed a two-stage procedure that is specially designed to find a system with the largest mean among those whose variances are smaller than a constant when the data under consideration are independent and identically distributed (IID) normal random variables. This two-stage procedure handles only a special case of our problem and can not be applied for a general stochastic constraint. Another problem with these procedures is that they become inefficient for 20 or more systems because they adopt Rinott's procedure-see Boesel et al. (2003) who explain where the inefficiency comes from.

Fully-sequential procedures take only a single basic observation from each alternative still in play at each stage of sampling and apply a decision strategy at every stage to eliminate apparently inferior alternatives early in the experimentation process. For this reason, such procedures are expected to reduce the overall simulation effort required to find the best system. Paulson (1964) and Hartmann (1988, 1991) presented fully sequential procedures in the case of IID normal data with equal variances, and Kim and Nelson (2001) extended their procedures in a direction to be more appropriate for simulation environments where 
it makes sense to assume both unequal variances across systems and dependence across systems due to the use of common random numbers (CRN). In this paper, we propose an efficient fully-sequential procedure that handles a general stochastic constraint.

This paper is organized as follows: In Section 2, we formulate the problem of interest. In Section 3, we present a procedure that finds a set of systems that satisfy a stochastic constraint. Then Section 4 gives a new procedure for the finding-the-best problem with a constraint. We present experimental results for the new procedure in Section 5, and conclude the paper with Section 6.

\section{PROBLEM}

In this section, we formulate our problem and define notation for the paper. We assume that output data from each system are IID normally distributed and that all systems are simulated independently; CRN are not considered in this paper. IID normality is plausible for example when the basic observations are either within-replication averages or batch means with a large batch size. Let $X_{i j}$ be an observation associated with a primary performance measure from replication $j$ of system $i$ and $Y_{i j}$ be an observation associated with a constraint (a secondary performance measure) from replication $j$ of system $i$. There are $k$ systems. The expected primary and secondary performance measures are defined as $x_{i}=E\left[X_{i j}\right]$ and $y_{i}=E\left[Y_{i j}\right]$, respectively. Then our problem amounts to finding

$$
\begin{array}{rc}
\operatorname{argmax}_{i=1, \ldots, k} & x_{i} \\
\text { s.t. } & y_{i} \leq Q,
\end{array}
$$

where $Q$ is a constant. (Of course, "greater than or equal to" constraints can be transformed to "less than or equal to" constraints by multiplying both sides by minus one.) We allow for dependence between $X_{i j}$ and $Y_{i j}$ because these random variables are likely to be correlated in practice. For instance, the throughput and downtime of a production system are usually negatively correlated.

For stochastic systems, it is not always possible to guarantee that we identify all systems satisfying the stochastic constraint. Instead we adopt an idea similar to that of the indifference-zone approach of Bechhofer (1954) to find a set of feasible or near-feasible systems. A decision maker will be asked to specify a range around the constant $Q$, say ( $Q_{\ell}, Q_{u}$ ) with $Q_{\ell}<Q_{u}$. Then three regions are defined:

- $y_{i} \leq Q_{\ell}$ : This is the desirable region. Any system in this range is feasible.

- $Q_{\ell}<y_{i}<Q_{u}$ : This is the acceptable region. It is possible that a feasible system in this range is declared infeasible and vice versa. We assume that one is willing to accept a system in this range as the best if it is declared "feasible" and our procedure chooses it as the best.

- $y_{i} \geq Q_{u}$ : This is the unacceptable region. A system in this range is infeasible and should be declined.

We can also define the following three sets for the constraint:

$S_{D}=$ the set of all desirable systems;

$S_{A}=$ the set of all acceptable systems;

$S_{U}=$ the set of all unacceptable systems.

For given $Q_{\ell}$ and $Q_{u}$, we define $q=\left(Q_{\ell}+Q_{u}\right) / 2$ and $\epsilon=\left(Q_{u}-Q_{\ell}\right) / 2$, respectively, and henceforth our procedures will be presented in terms of $q$ and $\epsilon$ rather than $Q_{\ell}$ and $Q_{u}$. Roughly speaking, $q$ is a target value that behaves as a cut-off point between acceptable and unacceptable systems and $\epsilon$ is a tolerance level that specifies how much we are willing to be below or above $q$.

In addition to $q$ and $\epsilon$, the decision maker needs to choose the indifference-zone parameter $\delta$ for the primary performance measure. This is the smallest absolute difference in the primary measure that the decision maker feels is important to detect. Thus, only "practical difference" matters, and we are indifferent among systems whose primary performance measures are within $\delta$ of the true best.

To solve the overall problem, we first identify a set of systems that contains all desirable systems, possibly with some acceptable systems (Phase I), and then we choose the best among the systems in that set (Phase II). Both steps need to be completed correctly with high probability. A procedure that can be used in Phase I is presented in Section 3. In Section 4, we propose procedure $\mathcal{A G K}$, where Phases I and II are performed sequentially-that is, Phase I is performed first and then completes Phase II is applied to the survivors from Phase I.

\section{A PROCEDURE FOR FEASIBILITY DETERMINATION}

In this section, we provide a procedure for Phase I that attempts to eliminate all unacceptable systems and to return a set consisting of all the desirable systems, possibly including some acceptable systems. 
First, we define some notation. Let $i \in\{1,2, \ldots, k\}$ and

$$
\begin{aligned}
n_{0}= & \text { initial first-stage sample size, } n_{0} \geq 2 ; \\
r= & \text { number of observations taken so far; } \\
S_{i}^{2}= & \text { the sample variance of } Y_{i j} \text { for } \\
& j=1,2, \ldots, n_{0} ; \\
W(r, v, w, z)= & \max \left\{0, \frac{v}{2}\left(\frac{w z}{v^{2}}-r\right)\right\}, \\
& \text { for any } v, w, z \in \mathbb{R} ; v, z \neq 0 .
\end{aligned}
$$

For all sets $A$, let $|A|$ denote the number of elements in $A$. Now we give an algorithm that finds a set of feasible systems.

\section{Algorithm I: Feasibility Determination Procedure}

Setup: $\quad$ Select $n_{0} \geq 2$ and nominal probability of correct selection (PCS) $1 / k<1-\alpha_{1}<1$. For a constraint, choose $\epsilon$ and $q$. Compute the constant

$$
\eta_{1}=\frac{1}{2}\left\{2\left[1-\left(1-\alpha_{1}\right)^{\frac{1}{k}}\right]^{-\frac{2}{n_{0}-1}}-1\right\} \text {. }
$$

Initialization: $\quad$ Let $R=\{1,2, \ldots, k\}$ and $F=\emptyset$ be the set of undetermined systems and the set of feasible systems, respectively. Let $h^{2}=2 \eta_{1}\left(n_{0}-1\right)$.

Obtain $n_{0}$ observations $Y_{i j}, j=1,2, \ldots, n_{0}$, from each system $i$. Compute $S_{i}^{2}$.

Set the observation counter $r=n_{0}$ and go to Feasibility Check.

Feasibility Check: For each system $i \in R$, if

$$
\sum_{j=1}^{r}\left(Y_{i j}-q\right) \leq-W\left(r ; \epsilon, h^{2}, S_{i}^{2}\right)
$$

then move $i$ from $R$ to $F$; else if

$$
\sum_{j=1}^{r}\left(Y_{i j}-q\right) \geq+W\left(r ; \epsilon, h^{2}, S_{i}^{2}\right)
$$

then eliminate $i$ from $R$.

Stopping Rule: If $|R|=0$, then return $F$ as the set of feasible systems.

Otherwise, take one additional observation $Y_{i, r+1}$ from each system $i \in R$, set $r=r+1$, and go to Feasibility Check.

Remark: Algorithm I can be performed without simulating systems simultaneously, so we can avoid the overhead of switching between systems - which is a typical disadvantage of fully-sequential procedures.
With high probability, the returned set $F$ will contain all desirable systems, plus possibly some acceptable systems, but no unacceptable systems. This event defines a correct selection (CS) for Algorithm I. More specifically, the following result is proved in Andradóttir, et al. (2005).

Theorem 1 Suppose that $Y_{i j}, j=1,2, \ldots$, are IID normally distributed, and $Y_{i j}$ and $Y_{\ell j}$ are independent for $i \neq \ell$. Then Algorithm I guarantees

$$
\operatorname{Pr}\{\mathrm{CS}\} \equiv \operatorname{Pr}\left\{S_{D} \subseteq F \subseteq\left(S_{D} \cup S_{A}\right)\right\} \geq 1-\alpha_{1}
$$

Algorithm I is closely related to the procedures for comparison with a standard presented by $\operatorname{Kim}(2003,2005)$. The comparison with a standard problem often turns up in the context of simulation (see Goldsman and Nelson 1998 for more details). In fact, the procedures due to Kim (2003, 2005) can be interpreted as special cases of Algorithm I.

\section{A PROCEDURE FOR COMPARING CONSTRAINED SYSTEMS}

In this section, we present procedure $\mathcal{A G K}$, where Phases I and II are performed sequentially. In particular, Algorithm I from Section 3 will be used in Phase I where we determine the feasibility of each system. For Phase II of $\mathcal{A G K}$, we adopt the Sequential Selection with Memory $(\mathcal{S S M})$ procedure due to Pichitlamken and Nelson (2001) and Pichitlamken et al. (2005). They extend the Kim and Nelson (2001) procedure for use within an optimization-viasimulation algorithm when simulation is costly and partial or complete information on alternatives previously visited is maintained. Since we assume that simulation is costly, we want to obtain $X_{i j}$ as well as $Y_{i j}$ when system $i$ is simulated in Phase $I$ and then retain those partial data for use in Phase II. However, saving $X_{i j}$ for later use in Phase II brings up a memory space issue, especially when Phase I is long and the number of survivors from Phase I is large. Procedure $\mathcal{S} \mathcal{S} \mathcal{M}$ requires saving only sample means and thus removes the memory space issue.

Before we present the new procedure, we need some more notation in addition to that defined in Section 3. In particular, let

$$
\begin{aligned}
S_{i \ell}^{2}= & \text { sample variance of the difference } X_{i j}-X_{\ell j} \\
& \text { between systems } i \text { and } \ell, j=1,2, \ldots, n_{0} ; \\
\bar{X}_{i}(r)= & \text { sample average of } X_{i j}, j=1,2, \ldots, r .
\end{aligned}
$$

The following $\mathcal{A G K}$ procedure simply applies Algorithm I first to find a set of feasible systems (Phase I) and then completes Phase II to find the best among the survivors from completed Phase I. 
Algorithm II: Procedure $\mathcal{A G K}$

Setup: Select the overall confidence level $1-\alpha$ and choose $\alpha_{1}$ and $\alpha_{2}$, where $\alpha_{1}+\alpha_{2}=\alpha$, for Phases I and II. Choose $\epsilon$ and $q$. Also select the indifference-zone parameter $\delta>0$ and firststage sample size $n_{0} \geq 2$. Calculate $\eta_{1}$ from Equation (1).

Initialization for Phase I: $\quad$ Let $R=\{1,2, \ldots, k\}$ and $F=\emptyset$ be the set of undetermined systems and the set of feasible systems, respectively. Let $h_{1}^{2}=$ $2 \eta_{1}\left(n_{0}-1\right)$.

Obtain $n_{0}$ observations $X_{i j}$ and $Y_{i j}, j=$ $1,2, \ldots, n_{0}$, from each system $i=1,2, \ldots, k$.

For all $i$ and $\ell \neq i$, compute the estimators $S_{i}^{2}$ and $S_{i \ell}^{2}$.

For each system $i$, compute $\bar{X}_{i}\left(n_{0}\right)$ and set $n_{i}=n_{0}$, the number of observations taken so far from system $i$ in Phase $I$.

Set the observation counter $r=n_{0}$ and go to Feasibility Check.

Feasibility Check: Same as in Algorithm I in Section 3. Stopping Rule for Phase I: If $|R|=0$, then

- if $|F|=0$, stop and return "no feasible system";

- if $|F|=1$, stop and return the system as the best;

- if $|F|>1$, go to Initialization for Phase II.

Otherwise, take one additional observation $X_{i, r+1}$ and $Y_{i, r+1}$ for each $i \in R$. Set $r=r+1$ and $n_{i}=n_{i}+1$ for each system $i \in R$. Then, update $\bar{X}_{i}(r)$ and go to Feasibility Check.

Initialization for Phase II: Set $m=|F|$. Compute

$$
\eta_{2}=\frac{1}{2}\left\{\left(\frac{2 \alpha_{2}}{m-1}\right)^{-\frac{2}{n_{0}-1}}-1\right\}
$$

and $h_{2}^{2}=2 \eta_{2}\left(n_{0}-1\right)$.

Let $R=F$ be the set of systems still in contention.

Set $r=n_{0}$ and go to Comparison.

Comparison: $\quad$ Set $R^{\text {old }}=R$. Let

$$
\begin{aligned}
R= & \left\{i: i \in R^{\text {old }}\right. \text { and } \\
& \sum_{j=1}^{r} \mathcal{X}_{i j} \geq \sum_{j=1}^{r} \mathcal{X}_{\ell j}-W\left(r ; \delta, h_{2}^{2}, S_{i \ell}^{2}\right), \\
& \left.\forall \ell \in R^{\text {old }}, \ell \neq i\right\}
\end{aligned}
$$

where

$$
\mathcal{X}_{i j}= \begin{cases}X_{i j} & \text { if } n_{i}<r \\ \bar{X}_{i}\left(n_{i}\right) & \text { if } n_{i} \geq r\end{cases}
$$

Stopping Rule for Phase II: If $|R|=1$, then stop and select the system whose index is in $R$ as the best. Otherwise, take one additional observation $X_{i, r+1}$ from each system $i \in R$ with $n_{i}<r$, and set $r=r+1$. Then go to Comparison.

The correct selection event for $\mathcal{A G K}$ is defined as the event of selecting a system $i$ such that $x_{i}>x_{[b]}-\delta$ and $i \in\left(S_{D} \cup S_{A}\right)$, where [b] is the identity of the system with the largest primary performance measure among the systems in $S_{D}$.

We have not yet been successful in proving the statistical validity of $\mathcal{A G K}$, although our experiments support its validity. However, Andradóttir et al. (2005) show that if one takes $m=k$ instead of $|F|$ in the Initialization for Phase II step of $\mathcal{A G K}$, then the procedure becomes statistically valid. However, this version of Algorithm II is less efficient than $\mathcal{A G K}$.

\section{EXPERIMENTAL RESULTS}

In this section we illustrate the performance of $\mathcal{A G K}$ based on experiments in which we use bivariate normal random variables for $\left(X_{i j}, Y_{i j}\right)$. We can assume that the target value $q$ for the secondary measure is $q=0$ without loss of generality. For simplicity, we also assume that no system is in the acceptable region. Therefore, all feasible systems are in the desirable region and all infeasible systems are in the unacceptable region. Let $f$ be the number of feasible systems and let system $f$ be the best feasible system. For replication $j$ of system $i=1,2, \ldots, k$, we assume that the vector $\left(X_{i j}, Y_{i j}\right)$ is bivariate normal with correlation $\rho$ and consider the following mean and variance configurations:

- We consider two mean configurations: The difficult means (DM) configuration and the monotonically increasing means (MIM) configuration. In the DM configuration,

$$
y_{i}= \begin{cases}-\epsilon, & i=1,2, \ldots, f, \\ \epsilon, & i=f+1, \ldots, k,\end{cases}
$$

and

$$
x_{i}= \begin{cases}0, & i=1,2, \ldots, f-1, \\ \delta, & i=f, \\ (i-1) \delta, & i=f+1, \ldots, k\end{cases}
$$

In DM, all feasible systems have $y_{i}$ exactly $\epsilon$ smaller than $q$ and all infeasible systems have $y_{i}$ 


\section{Andradóttir, Goldsman, and Kim}

exactly $\epsilon$ larger than $q$. Therefore, it is difficult to distinguish between feasible and infeasible systems. In addition, all feasible systems have $x_{i}$ very close to that of the true best feasible system, which makes it difficult to detect inferior feasible systems. All infeasible systems have much larger primary performance measures $x_{i}$ than that of the true best feasible system, which increases the chance for an infeasible system that is mistakenly declared feasible in Phase I to eliminate the true best feasible system. The DM configuration is used to test the validity of our new procedure under difficult circumstances.

To investigate the effectiveness of the procedures in eliminating infeasible or non-competitive systems, we use the MIM configuration. In the MIM configuration,

$$
y_{i}= \begin{cases}-(f-i+1) \epsilon, & i=1,2, \ldots, f \\ (i-f) \epsilon, & i=f+1, \ldots, k,\end{cases}
$$

and

$$
x_{i}=(i-1) \delta, \quad i=1, \ldots, k .
$$

In the MIM configuration, we only test the case where infeasible systems have larger $x_{i}$ than feasible systems. This is difficult, because if we fail to eliminate any infeasible system in Phase I, then the system is likely to be chosen as the best due to its large $x_{i}$, and this will increase the probability of an incorrect selection.

- We test only one variance configuration, namely constant variances. In particular, let $\sigma_{x_{i}}^{2}=\sigma_{y_{i}}^{2}=1$ denote the variances of the observations associated with the primary and secondary measures of system $i$, respectively. We refer readers to Andradóttir et al. (2005) for the performance of $\mathcal{A G K}$ with more variance configurations.

We test cases in which $k=5,15,25,101$ and $\rho=$ $-0.9,0,0.9$. We set $\delta=1 / \sqrt{n_{0}}$ and $\epsilon=1 / \sqrt{n_{0}}$. In all cases, we make 10,000 macro-replications. Our required confidence level is $1-\alpha=0.95$.

Our main results are as follows:

- Effect of Correlation: Correlation between observations of the primary and secondary performance measures seems to have very little effect on the performance of the procedures. Table 1 shows the estimated total number of replications (REP) and estimated PCS when there are twenty-five systems with thirteen feasible systems. As one can see, the REP and PCS do not change that much under various values of correlation $\rho$. We observed the same tendency for all configurations we tested. This is expected because the derivation of $\mathcal{A G K}$ is based on a Bonferroni-type inequality which dissolves the effect of dependence-although the procedure itself has not yet been proven to be statistically valid. Due to this insensitivity of the procedure to dependence, we will focus on the $\rho=0$ case only from now on.

Table 1: Average Total Number of Replications and Estimated Probability of Correct Selection for DM with $\rho=-0.9,0,0.9, k=25$, and $f=13$.

\begin{tabular}{||c|c||c|c||c|c||}
\hline \multicolumn{2}{||c||}{$\rho=-0.9$} & \multicolumn{2}{c||}{$\rho=0$} & \multicolumn{2}{c||}{$\rho=0.9$} \\
\hline \hline REP & PCS & REP & PCS & REP & PCS \\
\hline 4081 & 0.976 & 4063 & 0.973 & 3985 & 0.976 \\
\hline
\end{tabular}

- $\quad$ Performance of $\mathcal{A G K}$ : Table 2 shows the average total number of replications and estimated PCS when the DM and MIM configurations are employed with $f=1+(k-1) / 2, k=5,15,25,101$. The estimated PCS for the procedure is well over 0.95 for all DM configurations tested and is considerably larger under the MIM configuration. This is expected because it is much easier to detect infeasible and non-competitive systems under the MIM configuration. Table 2 also shows that $\mathcal{A G K}$ effectively eliminates infeasible and non-competitive systems under the MIM configuration.

Table 2: Total Number of Replications and Estimated PCS for $\mathcal{A G K}$ when $\rho=0$ and $f=1+(k-1) / 2$

\begin{tabular}{||c||c|c||c|c||}
\hline \multicolumn{1}{||c||}{} & \multicolumn{2}{c||}{ DM } & \multicolumn{2}{c||}{ MIM } \\
\hline$k$ & REP & PCS & REP & PCS \\
\hline \hline 5 & 576 & 0.969 & 459 & 0.981 \\
15 & 2233 & 0.971 & 1024 & 0.994 \\
25 & 4063 & 0.973 & 1387 & 0.996 \\
101 & 20566 & 0.974 & 3326 & 0.999 \\
\hline
\end{tabular}

\section{CONCLUSION}

In this paper, we consider a simple optimization problem with a single constraint. Our paper shows the possibility that R\&S procedures can be extended to solve more general discrete optimization problems with multiple constraints.

One can perform Phases I and II simultaneously instead of performing them sequentially as in $\mathcal{A G K}$. We refer to a procedure that performs Phase I and II simultaneously as $\mathcal{A G K}+$, and it is presented in Andradóttir et al. (2005) with proof of statistical validity. That paper also contains the full proof of Algorithm I, a statistically valid procedure that runs Phases I and II sequentially, and extensive experimental results. 


\section{Andradóttir, Goldsman, and Kim}

\section{ACKNOWLEDGMENT}

This material was supported by the National Science Foundation under Grant Number DMI-0400260. The first author was also supported by the National Science Foundation under Grant Numbers DMI-0000135 and DMI-0217860.

\section{REFERENCES}

Andradóttir, S., D. Goldsman, and S.-H. Kim. 2005. Fully sequential procedures for comparing constrained systems via simulation. Working Paper, School of Industrial and Systems Engineering, Georgia Institute of Technology, Atlanta, Georgia.

Bechhofer, R. E. 1954. A single-sample multiple decision procedure for ranking means of normal populations with known variances. Annals of Mathematical Statistics, 25:16-39.

Boesel, J., B. L. Nelson, and S.-H. Kim. 2003. Using ranking and selection to "clean up" after simulation optimization. Operations Research, 51:814-825.

Butler, J., D. J. Morrice, and P. W. Mullarkey. 2001. A multiple attribute utility theory approach to ranking and selection. Management Science, 47:800-816.

Chen, C.-H. 1996. A lower bound for the correct-selection probability and its application to discrete event simulations. IEEE Transactions on Automatic Control, 41:1227-1231.

Chen, H.-C., C.-H. Chen, L. Dai, and E. Yücesan. 1997. New development of optimal computing budget allocation for discrete event simulation. In Proceedings of the 1997 Winter Simulation Conference, ed. S. Andradóttir, K. J. Healy, D. H. Withers, and B. L. Nelson, 334341. Institute of Electrical and Electronics Engineers, Piscataway, New Jersey.

Chen, H.-C., C.-H. Chen, and E. Yücesan. 2000. Computing efforts allocation for ordinal optimization and discrete event simulation. IEEE Transactions on Automatic Control, 45: 960-964.

Chick, S. E. 1997. Selecting the best system: A decisiontheoretic approach. In Proceedings of the 1997 Winter Simulation Conference, ed. S. Andradóttir, K. J. Healy, D. H. Withers, and B. L. Nelson, 326-333. Institute of Electrical and Electronics Engineers, Piscataway, New Jersey.

Chick, S. E., and K. Inoue. 2001a. New two-stage and sequential procedures for selecting the best simulated system. Operations Research, 49:732-743.

Chick, S. E., and K. Inoue. 2001b. New procedures for identifying the best simulated system using common random numbers. Management Science, 47:1133-1149.

Goldsman, D., S.-H. Kim, W. S. Marshall, and B. L. Nelson. 2000. Ranking and selection for steady-state simulation. In Proceedings of the 2000 Winter Simulation
Conference, ed. J. Joines, R. R. Barton, P. Fishwick, and K. Kang, 544-553. Institute of Electrical and Electronics Engineers, Piscataway, New Jersey.

Goldsman, D., S.-H. Kim, W. S. Marshall, and B. L. Nelson. 2002. Ranking and selection procedures for steady-state simulation: Perspectives and procedures. INFORMS Journal on Computing, 14:2-19.

Goldsman, D., and B. L. Nelson. 1998. Comparing systems via simulation. In Handbook of Simulation: Principles, Methodology, Advances, Applications, and Practice, ed. J. Banks, 273-306. New York: John Wiley \& Sons.

Hartmann, M. 1988. An improvement on Paulson's sequential ranking procedure. Sequential Analysis, 7:363-372.

Hartmann, M. 1991. An improvement on Paulson's procedure for selecting the population with the largest mean from $k$ normal populations with a common unknown variance. Sequential Analysis, 10:1-16.

Kim, S.-H. 2003. Comparison with a standard via fully sequential procedures. In Proceedings of the 2003 Winter Simulation Conference, ed. S. E. Chick, P. J. Sanchez, D. Ferrin, and D. J. Morrice, 528-534. Institute of Electrical and Electronics Engineers, Piscataway, New Jersey.

Kim, S.-H. 2005. Comparison with a standard via fully sequential procedures. ACM TOMACS, 15:1-20.

Kim, S.-H., and B. L. Nelson. 2001. A fully sequential procedure for indifference-zone selection in simulation. ACM TOMACS, 11:251-273.

Kim, S.-H., and B. L. Nelson. 2005. On the asymptotic validity of fully sequential selection procedures for steady-state simulation. Operations Research, forthcoming.

Nelson, B. L., J. Swann, D. Goldsman, and W.-M. T. Song. 2001. Simple procedures for selecting the best system when the number of alternatives is large. Operations Research, 49:950-963.

Paulson, E. 1964. A sequential procedure for selecting the population with the largest mean from $k$ normal populations. Annals of Mathematical Statistics, 35:174-180.

Pichitlamken, J., and B. L. Nelson. 2001. A selection-ofthe best procedure for optimization via simulation. In Proceedings of the 2001 Winter Simulation Conference, ed. B. A. Peters, J. S. Smith, D. J. Medeiros, and M. W. Rohrer, 401-407. Institute of Electrical and Electronics Engineers, Piscataway, New Jersey.

Pichitlamken, J., B. L. Nelson, and L. J. Hong. 2005. A sequential procedure for neighborhood selection-of-thebest in optimization via simulation. European Journal of Operational Research, forthcoming.

Rinott, Y. 1978. On two-stage selection procedures and related probability-inequalities. Communications in Statistics, A7:799-811.

Santner, T. J., and A. C. Tamhane. 1984. Designing experiments for selecting a normal population with 
a large mean and a small variance. In Design of Experiments - Ranking and Selection: Essays in Honor of Robert E. Bechhofer, ed. T. J. Santner and A. C. Tamhane, 179-198. New York: Marcel-Dekker.

\section{AUTHOR BIOGRAPHIES}

SIGRÚN ANDRADÓTTIR is a Professor in the School of Industrial and Systems Engineering at the Georgia Institute of Technology. She received a B.S. in Mathematics from the University of Iceland in 1986, an M.S. in Statistics from Stanford University in 1989, and a Ph.D. in Operations Research from Stanford University in 1990. Her research interests include stochastic optimization, simulation, and stochastic processes. Her e-mail address is <salisye.gatech.edu $>$ and her web page is <www. isye.gatech.edu/faculty/sa/>.

DAVID GOLDSMAN is a Professor in the School of Industrial and Systems Engineering at the Georgia Institute of Technology. He received his Ph.D. in Operations Research and Industrial Engineering from Cornell University. His research interests include simulation output analysis and ranking and selection. He is an active participant in the Winter Simulation Conference, having been Program Chair in 1995, and having served on the WSC Board of Directors since 2002. His e-mail address is <sman@isye.gatech.edu $>$, and his web page is <www. isye.gatech. edu/ $\sim \operatorname{sman} />$.

SEONG-HEE KIM is an Assistant Professor in the School of Industrial and Systems Engineering at the Georgia Institute of Technology. She received her Ph.D. in Industrial Engineering and Management Sciences from Northwestern University in 2001. Her research interests include ranking and selection, quality control, and simulation output analysis. She is a member of INFORMS and IIE, and serves the editorial board of the Simulation Department of IIE Transactions. Her e-mail and web addresses are <skimaisye.gatech.edu> and <www. isye.gatech.edu/ skim/>, respectively. 